\title{
More or less is fine: an undercover work of DKK1 in anthrax toxin uptake
}

\author{
MAO Bing $\mathrm{Yu}^{1 *} \& \mathrm{WU} \mathrm{Wei}^{2}$ \\ ${ }^{1}$ State Key Laboratory of Genetic Resources and Evolution, Kunming Institute of Zoology, Chinese Academy of Sciences, Kunming 650223 , \\ China; \\ ${ }^{2}$ School of Life Sciences, Tsinghua University, Beijing 100084, China
}

Received May 2, 2014; accepted May 6, 2014; published online May 26, 2014

Citation: Mao BY, Wu W. More or less is fine: an undercover work of DKK1 in anthrax toxin uptake. Sci China Life Sci, 2014, 57: 735-736, doi: $10.1007 / \mathrm{s} 11427-014-4679-1$

The protective antigen (PA) mediated internalization of the anthrax complexes is critical in the development of anthrax, which depends on specific membrane receptors, namely ANTXR1 (also known as TEM8) and ANTXR2 (also known as CMG2) [1]. In a screen for host proteins required for anthrax toxin action, low-density lipoprotein receptor-related protein 6 (LRP6) was found important for efficient toxin entry in human M2182 prostate carcinoma cells [2], which interacts with both ANTXR1 and ANTXR2. However, the role of LRP6 in anthrax toxicity remains controversial as both in mice and in human HeLa cells, knockout or knockdown of LRP6 has no clear effect on their sensitivity to anthrax toxins [3].

LRP6 is an essential co-receptor for Wnt signaling, playing crucial roles in embryonic development, tissue homeostasis as well as tumorigenesis. LRP6 also serves as a co-receptor for the secreted Wnt inhibitor, Dickkopf1 (DKK1), which binds LRP6 and inhibits Wnt binding and signaling. The DKK1 binding triggers internalization of LRP6, especially in the presence of another DKK1 co-receptor, Kremen [4]. In the paper by Qian et al. [5], the authors explored the potential role of DKK1/LRP6/Kremen complex in anthrax toxin uptake in different cell lines.

In M2182 cells, DKK1 knockdown by shRNA and knockout by TALEN both reduced the sensitivity of cells to anthrax toxins. In the RAW264.7 macrophage cell line, ex-

*Corresponding author (email: mao@mail.kiz.ac.cn) posure of the cells to DKK1 antibodies also decreased the toxicity of anthrax toxin in a dose-dependent manner, suggesting that DKK1 promotes toxin uptake under these conditions. Mechanistically, they showed that DKK1 is in complex with ANTXR1 and ANTXR2 and facilitates internalization of anthrax toxins together with their receptors. Co-expression of Kremen with DKK1 and LRP6 further enhanced the uptake of anthrax toxins, highly reminiscent of the rapid internalization of the DKK1/LRP6/Kremen complex [4]. These results demonstrate that the anthrax toxins hijack the DKK1/LRP6/Kremen complex to mediate their entry into the target cells.

Interestingly, DKK1 does not simply promote the uptake of anthrax toxin; it functions in a bidirectional manner: moderate level of DKK1 promotes toxin uptake, while too low or too high level of DKK1 has little effect. In HeLa cells (which have a low endogenous DKK1 level) or the $D K K 1^{-1-}$ M2182 cells, overexpression of DKK1 with increasing doses firstly enhanced the sensitivity of the cells but then reduced it with higher DKK1 doses.

These data might suggest a simple model for the role of DKK1/LRP6 in anthrax toxin uptake (Figure 1): the DKK1/LRP6 complex promotes anthrax toxin uptake through promoting the co-internalization of the toxin-receptor complex, especially in the presence of Kremen. The effect of LRP6 on anthrax toxin uptake would be weak without DKK1 but become evident with moderate level of DKK1. With overloaded DKK1, however, another possibi- 


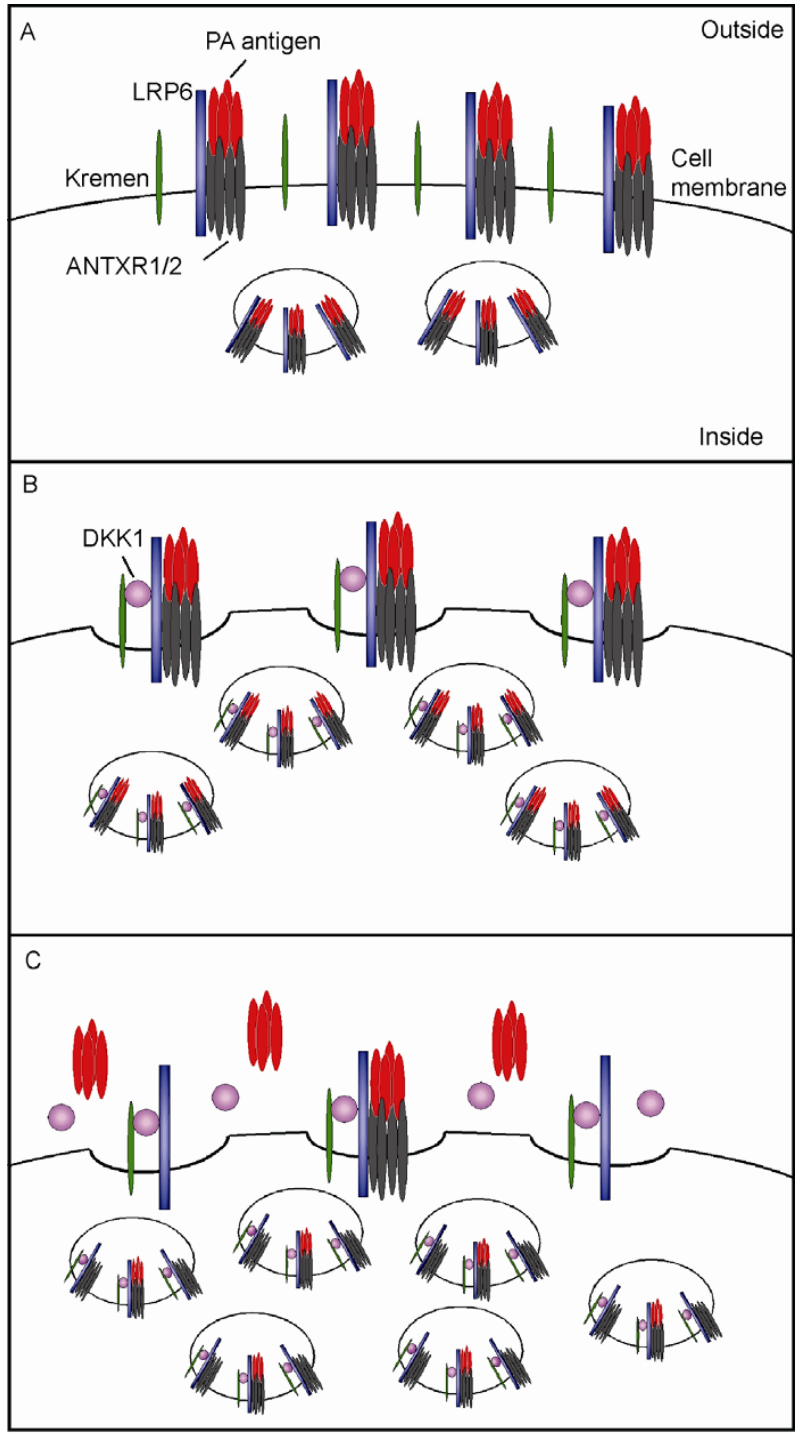

Figure 1 Models for the role of DKK1 in anthrax toxin uptake. A, In the absence of DKK1, the uptake of anthrax toxin mediated by ANTXR1/2 is kept at a low rate. B, Moderate level of DKK1 protein promotes rapid internalization of LRP6, especially in the presence of Kremen, carrying along the anthrax toxin-receptor complex. C, Overloaded DKK1, however, leads to clearance of LRP6 together with the ANTXRs, thus reduces toxin uptake. lity may exist that the membrane toxin receptors (ANTXR1 and ANTXR2) might be cleared from the cell surface due to their rapid endocytosis together with the DKK1/Kremen/ LRP6 complex, thus the toxin uptake gets limited. Such a model would propose that cells with high DKK1 and LRP6 expression show higher sensitivity to anthrax toxins, and in cells with low DKK1 expression, the role of LRP6 in anthrax toxin uptake is less prominent. Thus the difference of DKK1 expression levels might be the reason for the seemingly different role of LRP6 in anthrax toxicity in different cells. Of note, whether the endogenous expression of DKK1 would reach the inhibitory level still requires further investigation.

The current study suggests that the DKK1/LRP6/Kremen complex likely works as a Trojan horse to facilitate anthrax toxin uptake. The internalization of LRP6 and Kremen induced by DKK1, as well as that of the anthrax toxinreceptor complex, has been suggested to be clathrinmediated. However, the following fates of the internalized receptors are not well documented. In addition, Wnt3a has also been shown to induce internalization of LRP6 with caveolin, and whether Wnt3a and other Wnt ligands could promote anthrax toxin uptake is also an open question.

1 Young JA, Collier RJ. Anthrax toxin: receptor-binding, internalization, pore formation, and translocation. Annu Rev Biochem, 2007, 76: 243-265

2 Wei W, Lu Q, Chaudry GJ, Leppla SH, Cohen SN. The LDL receptor-related protein LRP6 mediates internalization and lethality of anthrax toxin. Cell, 2006, 124: 1141-1154

3 Young JJ, Bromberg-White JL, Zylstra C, Church JT, Boguslawski E, Resau JH, Williams BO, Duesbery NS. LRP5 and LRP6 are not required for protective antigen-mediated internalization or lethality of anthrax lethal toxin. PLoS Pathog, 2007, 3: e27

4 Mao B, Wu W, Davidson G, Marhold J, Li M, Mechler BM, Delius H, Hoppe D, Stannek P, Walter C, Glinka A, Niehrs C. Kremen proteins are dickkopf receptors that regulate $\mathrm{Wnt} /$ beta-catenin signalling. Nature, 2002, 417: 664-667

5 Qian L, Cai C, Yuan P, Jeong SY, Yang X, Almeida VD, Ernst J, Costa M, Cohen SN, Wei W. Bidirectional effect of Wnt signaling antagonist DKK1 on the modulation of anthrax toxin uptake. Sci China Life Sci, 2014, 57: 469-481

Open Access This article is distributed under the terms of the Creative Commons Attribution License which permits any use, distribution, and reproduction in any medium, provided the original author(s) and source are credited. 